\title{
A microscopic look at the reinforcement of silica-filled rubbers
}

\author{
A. Botti \\ Dipartimento di Fisica “E. Amaldi,” Universitá Roma TRE, I-00146 Roma, Italy \\ W. Pyckhout-Hintzen a) and D. Richter \\ IFF-Forschungszentrum Jülich, D-52425 Jülich, Germany \\ V. Urban \\ Chemical Sciences Division, ORNL, Oak Ridge, Tennessee 37831-6100 \\ E. Straube \\ FB Physik, Martin-Luther-Universität Halle-Wittenberg, D-06099 Halle, Germany
}

(Received 21 December 2005; accepted 7 March 2006; published online 5 May 2006)

\begin{abstract}
The deformed structure of silica-filled elastomers under uniaxial strain has been investigated using a combination of both small angle x-ray scattering and small angle neutron scattering methods. Using an extraction procedure and taking into account the two-phase nature of these polymer-based composites, the single chain scattering behavior as well as filler properties could be obtained uniquely on identical samples. For the first time the deformation of the rubbery matrix on the length scale of the network chain in a filled rubber could be determined and therewith the importance of matrix overstrain for the mechanical properties was estimated. Additionally, the determination of filler deformation and filler destruction presents microscopic details of the mechanisms of filler networking and the stress-softening Mullins effect. (C) 2006 American Institute of Physics.
\end{abstract}

[DOI: 10.1063/1.2191048]

\section{INTRODUCTION}

The field of composite materials is a fast-growing branch of modern polymer physics ${ }^{1-13}$ and very strongly empirically based and technically guided for which a firm molecular basis is yet to be provided. The understanding of material properties especially of reinforcement in these heterogenous composites is intimately related to the structure of and the interplay between the constituent components. ${ }^{6,8,14}$ The mechanical response of filled elastomers has been the object of many studies already and a benchmark for a number of theories. ${ }^{15-17}$ Even though the mechanical properties are always determined by a superposition of several processes, in such systems the complex dependence on deformation and deformation history allows to a certain extent the separation of mechanisms. For example, the observed amplitude and time dependency of dynamical properties necessitated the introduction of filler networking and also the reexamination of the role of filler-filler and chain-filler interactions in explaining the Mullins effect. ${ }^{12,16,17}$ Consequently, the concept of matrix overstrain in networks with colloidal highly structured fillers as expressed by micromechanical or phenomenological treatments resulting in expressions such as $E$ $=E_{m} F\left(\phi_{\text {eff }}\right)$ for the modulus or $\sigma=\sigma_{m}\left(\lambda^{*}\right)$ for the stress had to be revised. Here, $\lambda^{*}$ is the enhanced average local deformation of the matrix and the subscript $m$ denotes matrix properties. $\phi_{\text {eff }}$ is the effective volume fraction of the filler taking into account all less deformable parts of the rubber matrix (for details see, e.g., Ref. 16). One of the typical

${ }^{a)}$ Electronic mail: w.pyckhout@fz-juelich.de expressions is the Pade approximation of the second order power series for a polydisperse system of hard spheres ${ }^{3}$

$$
F(\phi)=1+\frac{2.5 \phi_{\mathrm{eff}}}{1-2 \phi_{\mathrm{eff}}},
$$

which is demonstrated in Fig. 1. Expressions like Eq. (1) were considered to reflect the physics of the reinforcement of filled rubbers in virgin state at small deformations quite reasonably. The effective volume fraction which is the only necessary free parameter was found to be compatible with the large pore volume which may be occupied by immobilized matrix material within highly structured fillers. As already mentioned above the typical hysteresis at cyclic deformations and the "return" to the virgin curve at further increasing deformations required the introduction of filler network con-

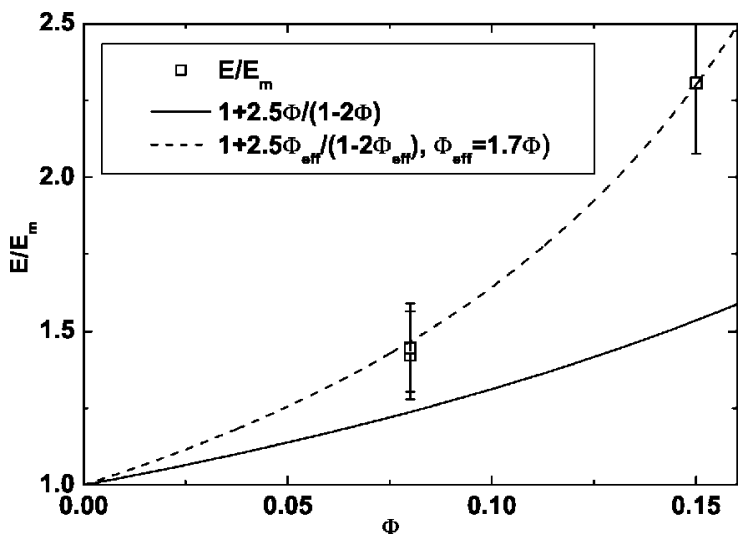

FIG. 1. Relative modulus increase and description by strain amplification according to Eq. (1). Data points refer to all samples, specified in Table I. 
tributions and therewith decreasing contributions from the matrix and consequently less overstrain. Recent results obtained by deuterium NMR yield an even more radical picture and found no indication of overstrain at all in large parts of the matrix. ${ }^{18}$ On the other hand, overstrain could unambiguously be detected in a model latticelike microphase-separated system. $^{19}$

Taking into consideration these contradictive results the ability of small angle neutron scattering (SANS) to observe local matrix deformations on the length scale of a polymer chain as well of the filler phase becomes highly challenging. With SANS both selected labeling and contrast matching allow to highlight special structures and make it possible to attempt a direct observation of the proposed mechanisms. The impossibility of complete contrast matching for systems with colloidal fillers such as carbon black or silica will be overcome by small angle x-ray scattering (SAXS) and SANS investigations on the same systems. In this investigation we will therefore focus on the deformation behavior of the rubbery matrix as well as of the filler system itself on a microscopic scale for a model system for silica-filled rubbers.

\section{SCATTERING ANALYSIS}

In a preceding publication on the characterization of typical multicomponent structures with $\mathrm{x}$ rays and neutrons, ${ }^{20}$ a general scattering theory was developed and applied to systems in the isotropic state. The approach solely assumed that the system consists of two incompressible phases (filler and polymer matrix) and that the distribution of the polymer chains over the polymer phase is not influenced by the presence of the filler. This corresponds to the fact that for the given system no preferential interactions of the polymer with the filler phase should be expected. For a threecomponent blend, i.e., filler particles with constant scattering properties and a deuterated/protonated blend as rubber phase, the differential scattering cross section resulted in an apparently incoherent addition of two terms,

$$
\frac{d \sigma}{d \Omega}(q)=\left(b_{f}-b_{\langle P\rangle}\right)^{2} S_{f}(q, \lambda)+\phi_{P}\left(b_{\mathrm{H}}-b_{\mathrm{D}}\right)^{2} S_{\mathrm{RPA}, P}\left(q, \lambda_{m}\right),
$$

where $P$ and $f$ stand for polymer chains and embedded filler particles, respectively, with scattering lengths $b_{f}, b_{\mathrm{H}}, b_{\mathrm{D}}$, and $b_{\langle P\rangle}$ for the filler, the protonated and deuterated chains, and their average. $S_{f}(q, \lambda)$ is the structure factor of the filler system, and $S_{\mathrm{RPA}, P}\left(q, \lambda_{m}\right)$ is the RPA-structure factor of the polymer matrix at the local deformation $\lambda_{m}$. From this it is clear that single chain properties are ideally obtained if matching of the filler, i.e., cancelling the first term, was achieved. Exactly this parasitic scattering $\left(\simeq S_{f}\right)$ is obtained ideally from SAXS measurements on the very same sample which simply sees $S_{f}$ due to vanishing contrast, i.e., the second term between deuterated and protonated chains. The strict additivity of phase and intrapolymer scattering according to Eq. (2) was proved in Ref. 20 using the $q$ dependence of $S_{f}$ to obtain the chain scattering from the polymer matrix also for nonideal matching. Extending this concept to a deformed system of cross-linked and entangled chains our
TABLE I. Composition of the samples. $\phi_{\mathrm{D}}$ is the fraction of labeled polymer in the rubbery phase, $\phi$ the volume fraction of filler 6105 , and $E / E_{m}$ the increase of the modulus in comparison to the rubber matrix.

\begin{tabular}{lccccc}
\hline \hline Sample & $\phi_{\mathrm{D}}$ & $\phi_{\mathrm{H}}$ & $\phi$ & $M_{c}(\mathrm{~g} / \mathrm{mol})$ & $E / E_{m}$ \\
\hline 5 C99 & 0.40 & 0.60 & 0.08 & 12500 & 1.42 \\
7 C99 & 0.34 & 0.66 & 0.08 & 12000 & 1.45 \\
6 C99 & 0.40 & 0.60 & 0.15 & 12800 & 2.31 \\
\hline \hline
\end{tabular}

well- proved approach for networks made from long primary chains will be applied to $S_{\mathrm{RPA}, P}\left(q, \lambda_{m}\right)$. $S_{\mathrm{RPA}, P}\left(q, \lambda_{m}\right)$ can be expressed by the product of the RPA-structure factor $S_{0 \mathrm{RPA}, P}\left(q, \lambda_{m}\right)=S_{0, \mathrm{RPA}, P}\left(q \lambda_{m}\right)$ of the deformed tube axes, ${ }^{21}$

$$
S_{0, \mathrm{RPA}, P}\left(q \lambda_{m}\right)=\frac{\phi_{\mathrm{H}} \phi_{\mathrm{D}} N_{w}}{1-2 \chi N_{w} P_{0}\left(q \lambda_{m}\right) \phi_{\mathrm{H}} \phi_{\mathrm{D}}} P_{0}\left(q \lambda_{m}\right),
$$

and the contribution from restricted fluctuations modeled by the Warner-Edwards tube-model structure factor,

$$
\begin{aligned}
P_{W E}\left(\mathbf{q}, \lambda_{m}\right)= & 2 \int_{0}^{1} d \eta \int_{0}^{\eta} d \eta^{\prime} \prod_{\mu} \exp \left(-\left(q_{\mu} R_{g}\right)^{2}\right. \\
& \times\left[\lambda_{m, \mu}^{2}\left(\eta-\eta^{\prime}\right)-\left(1-\lambda_{m, \mu}^{2}\right) \frac{d_{\mu}^{2}}{2 \sqrt{6} R_{g}^{2}}\right. \\
& \left.\left.\times\left(1-\exp \left(-\left(\eta-\eta^{\prime}\right) \frac{2 \sqrt{6} R_{g}^{2}}{d_{\mu}^{2}}\right)\right)\right]\right),
\end{aligned}
$$

normalized by the bare structure factor $P_{0}\left(q \lambda_{m}\right)$ of the labeled chains as

$$
S_{\mathrm{RPA}, P}\left(q, \lambda_{m}\right)=S_{0, \mathrm{RPA}, P}\left(q, \lambda_{m}\right) P_{W E}\left(\mathbf{q}, \lambda_{m}\right) / P_{0}\left(q \lambda_{m}\right) .
$$

The deformation ratio $\lambda_{m}$ is the deformation on the length scale of the labeled primary chains, $N, \chi$, and $\phi$ are polymerization degree, Flory-Huggins interaction parameter, and volume fractions. $R_{g}, q_{\mu}$, and $d_{\mu}=d_{0} \lambda_{m}^{1 / 2}$ are the radius of gyration and the components of the scattering vector and the fluctuation range of the chains. $\eta$ and $\eta^{\prime}$ are dimensionless integration variables over the chain contour.

\section{EXPERIMENT}

The preparation of the matrix rubbers was described already in detail in the former publication to which we refer ${ }^{20}$ and is only summarized here. Model polyisoprene (PI) homopolymers were synthesized through living anionic polymerization. The final products were deuterated D-PI $\left(M_{w}\right.$ $=196000, P=1.019, \mathrm{H}$ equivalent $)$ and protonated H-PI $\left(M_{w}=206000, P=1.020\right)$. The polydispersity index $P$ $=M_{w} / M_{n}$ was determined by SEC and $M_{w}$ from independent LALS. The TESPT-organosilane-modified filler Coupsil 6105 (Degussa AG) with 5\% surface activation was based on the parent Ultrasil VN2 and used as received. Three samples which differed in labeling degree were prepared by solution blending, ultrasound treatment, and evaporation under high vacuum with varying filler volume fraction. Their compositions are listed in Table I. As the bulk density of the used silica, $2.0 \mathrm{~g} / \mathrm{cm}^{3}$, is only known to within $10 \%$ accuracy and also irregularities in the surface modifications may contrib- 
ute, the contrast matching of silica (i.e., scattering length density $b / V_{\mathrm{SiO}_{2}}=\rho_{\mathrm{SiO}_{2}}=3.1 \times 10^{10} \mathrm{~cm}^{-2}$ ) with a blend of polyisoprene cannot be achieved ideally, and therefore blends with composition close to or around the expected contrast matching composition $\left(\phi_{\mathrm{D}}=0.43\right)$ were prepared. The cross-linking process was random using dicumylperoxide as described. The cross-link density was estimated from swelling degree measurements in cyclohexane. We use the concepts developed by Berriot et al. $^{22}$ to relate the measured swelling ratios $Q_{0}$ of the filled systems to the swelling ratios $Q_{\text {pol }}$ of a polymer matrix with the same cross-link and entanglement density. Our systems show comparable swelling ratios as the systems of Beriot. Therefore, the same relationships were adopted for $Q_{0} / Q_{\text {pol }}$ to be 0.8 and 0.65 , respectively, for $\phi=0.08,0.15$. Using the usual Flory-Rehner expression $^{23}$ the molecular weights between the cross-links $M_{c}$ were determined and are given in Table I. The modulus of the matrix was estimated using data for similar networks with mesh sizes between 3000 and $16000 \mathrm{~g} / \mathrm{mol}$, investigated by swelling and uniaxial stress-strain analysis. From the reported chemical cross-link contribution $G_{c} \sim M_{c}^{-1}$ and entanglement contribution $G_{n} \sim M_{c}^{0}$, a regression formula $E_{m} / M P a=0.105+5.73 /\left(M_{c} / \mathrm{kg} \mathrm{mol}\right)$ results. $^{24}$

The Young modulus of the filled rubbers was determined by stress-strain tests on a tensile testing unit (Instron 4443) at constant low crosshead speed $(1 \mathrm{~mm} / \mathrm{min}, 0.02 / \mathrm{min}$, and $\epsilon_{\max }=0.2$ on the same samples after the scattering investigations). The deformation ratio was determined from the clamping displacement and the initial length of the samples. The ratio of moduli $E / E_{m}$ with the matrix modulus $E_{m}$ determined as described above is shown in Table I and Fig. 1 together with predictions according to Eq. (1). The resulting effective filling degree $\phi_{\text {eff }}=1.7 \phi$ affirms that the investigated samples possess the typical features of carbon black or silica-filled rubbers.

SANS data on the deformed elastomers as prepared were obtained from the small angle neutron diffractometer (SAND), Argonne National Laboratories, Chicago and KWS1, FZ Jülich. Synchroton SAXS measurements were performed after this at ID2, ESRF, Grenoble. Small strain steps, compared to the discrete larger values in the neutron experiments, could be achieved due to the high photon flux. The common scattering vector range $q$ of both scattering experiments was about 0.002 up to $0.2 \AA^{-1}$. All data were obtained two dimensionally and corrections for detector sensitivity, solid angle, and the usual background subtractions applied channelwise before reducing the data corresponding to principal axis directions. This was necessary in view of the different type of data, obtained from a steady source (KWS1,ID2) and a pulsed source (SAND). Parallel and perpendicular components of the scattering relative to the stretching direction were determined from narrow sector opening angles.

\section{EVALUATION OF SCATTERING DATA}

For the determination of the matrix deformation from scattering data using the theory outlined above, $S_{f}(q, \lambda)$ in the deformed state must be known. Figure 2 shows the re-

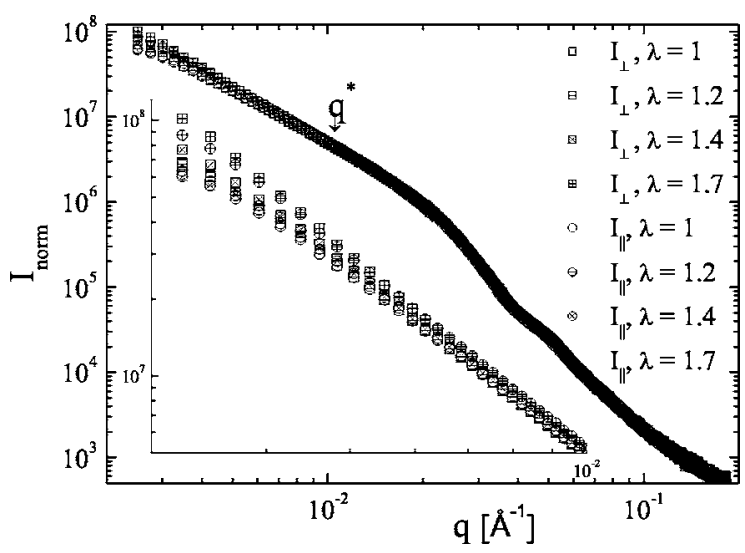

FIG. 2. SAXS-data for sample 6C99. The insert shows the data for $q<q^{*}$ $\simeq 10^{-2} \AA^{-1}$

sults of SAXS measurements for sample 6C99 with $\phi$ $=0.15$. It is eminent that for this and also all other investigated samples above a certain $q$ value $\left(q^{*}\right)$ the scattering is independent of the deformation ratio of the sample. Latter affects only the smallest $q$ systematically and will be discussed below. For the treatment of the SANS data it is of importance that $S_{f}(q, \lambda)$ is $\lambda$ invariant over the experimental $q$ range and consequently depends on $|\mathbf{q}|$ only. The phase scattering then drops out for any difference of SANS intensities with the same $|\mathbf{q}|$. Therefore, fitting the difference of SANS intensities parallel and perpendicular to the deformation direction, only the description of matrix data according to Eqs. (2)-(5) enters the corresponding procedures. In the more general case of an anisotropic filler scattering the $\mathbf{q}$ dependence of $S_{f}$ must be fed in and only a scaling factor relating SAXS and SANS scatterings from the filler has to be determined in the same way as we proposed in the isotropic characterization study of our system. Figure 3 shows the results of such a description of the SANS data using Eqs. (3)-(5) for the sample 5C99 with $\phi=0.08$. This example should exhibit the most clear features as the investigated strain was the highest one in this study. Before discussing the results and details of the fitting procedure of the SANS data the attention should be emphasized on the result depicted here. The isotropic phase scattering extracted from the scattering along the two main axes is compared with the phase

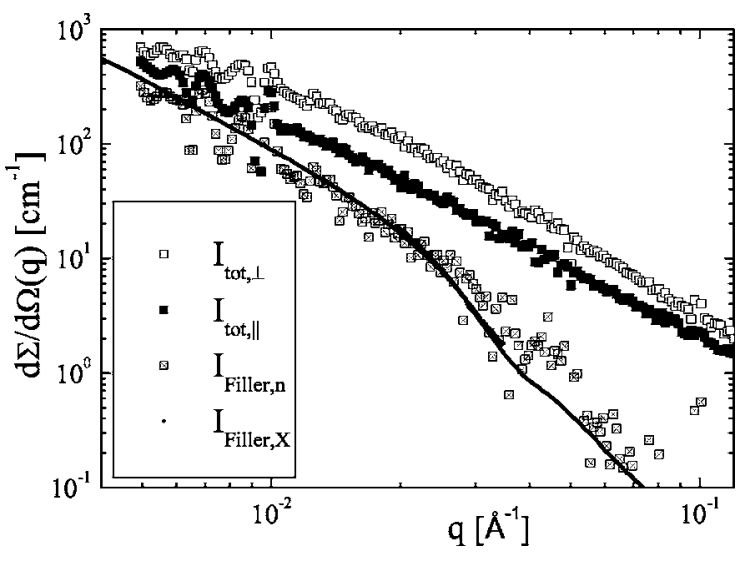

FIG. 3. SANS-data for sample 5C99, extracted phase scattering, and rescaled SAXS data for $\lambda=1.8$. 
(a)

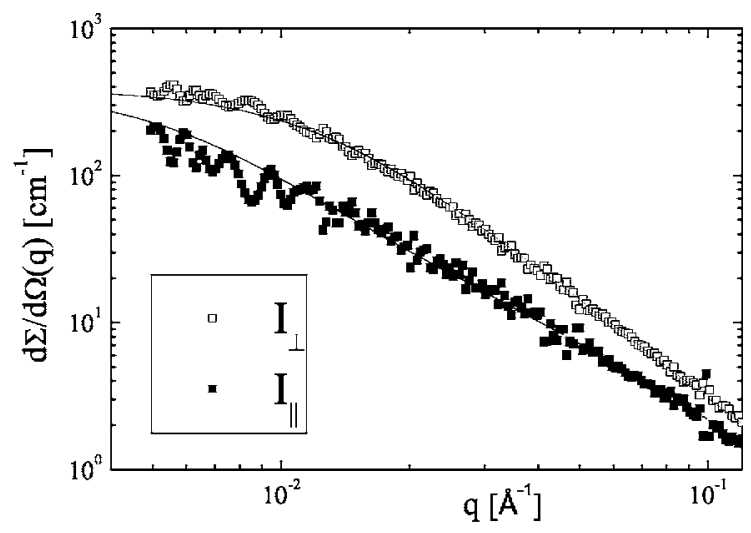

(b)

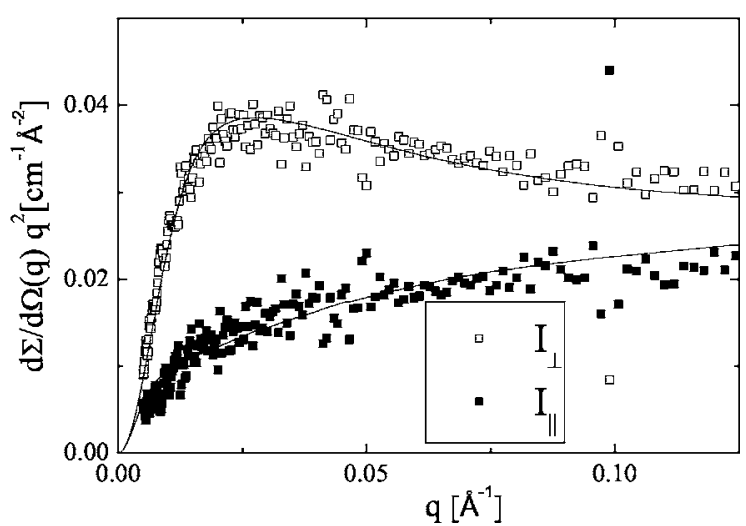

FIG. 4. Extracted chain scattering of sample 5C99 and theoretical description according to Eqs. (3)-(5) in log-log and Kratky representation for $\lambda=1.8$.

scattering from SAXS on the same sample. The agreement is strikingly good and confirms the suggested approach. The scatter at high $q$ is due to intensity levels which submerse in the incoherent background. The results for the resulting polymer scattering and the theoretical data according to Eqs. (3)-(5) are presented in Fig. 4. An excellent agreement especially for the very sensitive Kratky plot can be noticed.

The main fitting parameter was the deformation ratio of the matrix $\lambda_{m}$ whereas all other parameters of Eq. (4) were fixed or taken from the isotropic state. Additionally, the intensity $I_{0, \text { th }}$ at $q=0$ and the background $u$ were fitted to avoid undesirable influences from the limited accuracy of the calibration of the data. $R_{g}$ of the chain was evaluated as $146 \AA$ from the isotropic data, $\chi$ is estimated as $\sim 2 \times 10^{-4}$. The values of the parameters are given in Table II and in the corresponding table caption. The obtained deformation ratio is quasi-identical to the macroscopic deformation of the sample and no sign of overstrain can be observed therewith on the chain level. To facilitate the comparison with the strain amplification factor $F(\phi)$, the corresponding quantity $F_{n}=\left(\lambda_{m}-1\right) /(\lambda-1) \sim 1$ was introduced. The values for $F_{n}$ presented in Table II are close to 1 where the errors are determined mainly by the accuracy of the applied macroscopic strain which is about 0.1 . The overall uncertainty including those from the fitting procedure is about 0.15 . Strain amplifications of the order given by the modulus enhancement are outside of the range of errors and would lead to much too strong anisotropic patterns than observed experimentally. The found $F_{n}$ values which are only slightly lower than 1 are then most probably due to the overestimation of the deformation ratio in the scattering experiment. From the overall good agreement of the theoretical description and the experimental data it can also be concluded that the deformation is rather homogeneous, e.g., the data exclude very sensitively the presence of more than $10 \%$ of undeformed (immobilized) or also higher deformed rubber in addition to the affinely stretched chains. To validate this result it should be pointed out that for the tube radius $d_{\mu}$ in Eq. (4) the result obtained from unfilled networks $d_{0}=42 \AA$ was used. It is necessary to fix $d_{0}$ since the deformation dependence of $P_{W E}\left(\mathbf{q}, \lambda_{m}\right)$ according to Eq. (4) is sensitive to $d_{0}$ especially at larger $q$. On the other hand a property as $d_{0}=42 \AA$ with a length scale clearly below the scale of the filler particles should be unaffected by their presence.

The main parameters of the filler system were already determined in Ref. 20 for the undeformed system by a twolevel Beaucage-type analysis. ${ }^{25}$ The results are the size of the primary particles $R_{p}$, the radius of gyration of the cluster $R_{g, \mathrm{cl}}$ of $z$ primary particles, and two fractal dimensions $d_{p}$ and $d_{f}$ characterizing the particle surface and the cluster structure. Here, the same analysis applies with $R_{p}$ and $d_{p}$ fixed to the isotropic values in agreement with the $\lambda$ independence of the SAXS data at large $q$ and the expectation that primaryparticle related properties should be unchanged at deformation. The number of primary particles $z$ in a cluster and its fractal dimension $d_{f}$ are the only deformation-dependent parameters to describe the SAXS data at low $q$ in Fig. 2. The relations between the prefactors of the components of the Beaucage model were expressed by the usual relations as in Refs. 20 and 25. Figure 5 shows the results of two representative samples for the components of the radius of gyration of the clusters parallel and perpendicular to the direction of the deformation given by

$$
R_{g, \mathrm{cl}}=\sqrt{\left(2 R_{p}\right)^{2} z^{2 / d_{f} /\left(\left(1+2 / d_{f}\right)\left(2+2 / d_{f}\right)\right)},}
$$

for $\phi=0.08$ and 0.15 . The mass fractal exponents $d_{f}$ of the clusters increase slightly both parallelly and perpendicularly within the range of scatter. We note that according to $\phi^{*}$

TABLE II. Neutron scattering results from the extraction procedure for the effective three-component model. The absolute calibration of the data is in close agreement with expected intensities, given uncertainties in $\phi, \phi_{\mathrm{D}}$, and $M_{w} . F_{n}=\left(\lambda_{m}-1\right) /(\lambda-1)$.

\begin{tabular}{lccccccc}
\hline \hline Sample & $\phi$ & $\lambda$ & $I_{0, \mathrm{th}}\left(\mathrm{cm}^{-1}\right)$ & $I_{0, \mathrm{fit}}\left(\mathrm{cm}^{-1}\right)$ & $u\left(\mathrm{~cm}^{-1}\right)$ & $F_{n}$ & $F(\phi)$ \\
\hline 5C99 & 0.08 & $1.4,1.6,1.8$ & 336 & $264 \pm 23$ & $0.32 \pm 0.02$ & $0.89 \pm 0.15$ & 1.42 \\
7C99 & 0.08 & 1.2 & 305 & $275 \pm 10$ & $0.33 \pm 0.01$ & $0.83 \pm 0.15$ & 1.45 \\
6 C99 & 0.15 & 1.35 & 323 & $299 \pm 11$ & $0.34 \pm 0.02$ & $0.86 \pm 0.15$ & 2.3 \\
\hline \hline
\end{tabular}


(a)

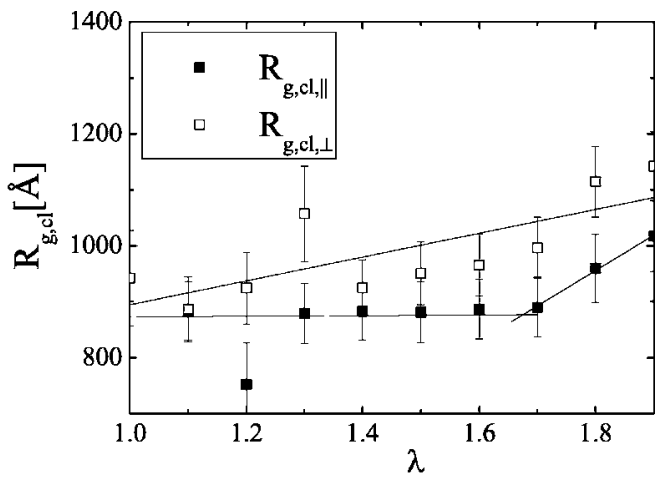

(b)

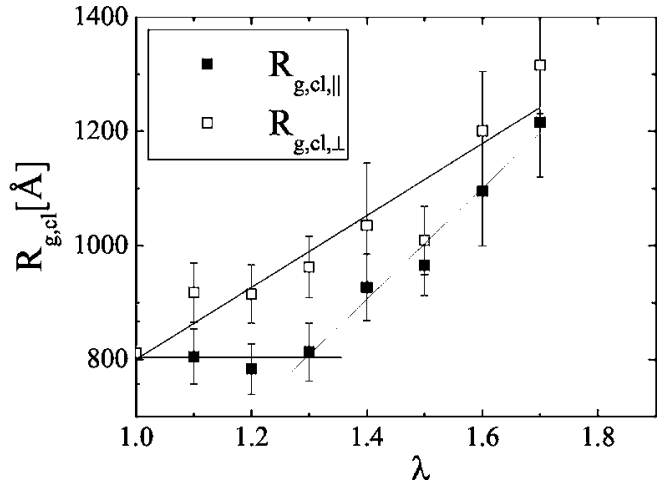

FIG. 5. $R_{g, \mathrm{cl}}$ for samples 5C99 and 6C99, respectively, $\phi=0.08$ and $\phi=0.15$

$\approx\left(R_{g, \mathrm{cl}} / R_{p}\right)^{d_{f}-3} \approx(900 / 124)^{d_{f}-3} \approx 0.2$, all investigated systems are below the overlap concentration and therefore the properties of single clusters should be reflected by $R_{g, \mathrm{cl}}$. In Fig. 5 for two different filler loadings $R_{g, \mathrm{cl}, \|}$ is constant at small deformations and increases almost proportional to $\lambda$ above a certain threshold whereas $R_{g, \mathrm{cl}, \perp}$ increases almost linearly with the deformation. In the simplest interpretation the transition from constant $R_{g, \mathrm{cl}, \|}$ to linear growth is assigned to the onset of cluster break which has been postulated only recently as the mechanism of the Mullins effect. ${ }^{12,16,17}$ This is a first microscopic proof of this process. This picture is also supported by the observed relationship of the deformations at the transition for the different filler loadings which correspond to nearly constant values of $E / E_{m}$ in Fig. 1, respectively. ${ }^{13}$ This suggests a loading-invariant stress at break of the clusters. The continuous increase of $R_{g, \mathrm{cl}, \perp}$ must be attributed to the fusion of clusters due to the reduction of perpendicular dimensions at uniaxial deformation. The dependence of the mass fractal exponents $d_{f}$ of the clusters on strain corroborates the increase of $R_{g, \mathrm{cl}, \perp}$ by cluster fusion which is accompanied by more compact clusters. ${ }^{13}$

\section{CONCLUSIONS}

To conclude, SANS measurements of partially labeled silica-filled rubbers in the nonmatching state have offered an independent tool to focus on both matrix and filler interactions. These first results on the rubbery phase for our model system show a clear failure of the still popular overstrain picture of rubber reinforcement and support the important role of filler networking. It should be noticed that the solution blending to achieve the model silica-filled rubbers were sufficiently close to typical rubbers with industrial importance. Although the analyzed network chain densities as well as the filling degree are about half the ones of representative rubbers, nevertheless the same typical properties could be identified. Further, this work also allowed the first direct observation of breaking-up processes in the filler system. The presented analysis was facilitated by the deformation independence of the filler scattering within the range of chain scattering but the presented method should be confidently applicable to more complex filled systems.

\section{ACKNOWLEDGMENTS}

The authors thank M. Hintzen for the preparation of the samples. Degussa AG is kindly thanked for providing the filler. The authors appreciate the comments of Professor G. Heinrich, IPF, Dresden and Dr. St. Westermann, Goodyear SA, Colmar-Berg.

${ }^{1}$ S. Ahmed and F. Jones, J. Mater. Sci. 25, 4933 (1990).

${ }^{2}$ B. Boonstra, Polymer 20, 691 (1979).

${ }^{3}$ R. Christensen, Mechanics of Composite Materials (Wiley, New York, 1979).

${ }^{4}$ S. Wolff and J. Donnet, Rubber Chem. Technol. 63, 32 (1990).

${ }^{5}$ G. Raos, Macromol. Theory Simul. 12, 17 (2003).

${ }^{6}$ M. Klueppel, R. Schuster, and G. Heinrich, Rubber Chem. Technol. 70, 243 (1996).

${ }^{7}$ G. Raos and G. Allegra, J. Chem. Phys. 113, 7554 (2000).

${ }^{8}$ M. Klueppel and J. Schramm, Macromol. Theory Simul. 9, 742 (2000).

${ }^{9}$ G. Huber and T. Vilgis, Kautsch. Gummi Kunstst. 52, 102 (1999).

${ }^{10}$ G. Heinrich, M. Klueppel, and T. Vilgis, Curr. Opin. Solid State Mater. Sci. 6, 195 (2002).

${ }^{11}$ T. Vilgis, Polymer 46, 4223 (2005).

${ }^{12}$ A. Lapra, F. Clement, L. Bobobza, and L. Monnerie, Rubber Chem. Technol. 76, 61 (2002).

${ }^{13}$ T. A. Witten, M. Rubinstein, and R. Colby, J. Phys. II France 3, 367 (1993).

14 A. Medalia, Rubber Chem. Technol. 60, 45 (1987).

${ }^{15}$ G. Kraus, Reinforcement of Elastomers (Wiley, New York, 1965).

${ }^{16}$ M. Klueppel, Adv. Polym. Sci. 164, 1 (2003).

${ }^{17}$ G. Heinrich and M. Klueppel, Adv. Polym. Sci. 160, 1 (2002).

${ }^{18}$ P. Ekanayake, H. Menge, H. Schneider, M. Ries, M. Brereton, and P. G. Klein, Macromolecules 33, 1807 (2000)

${ }^{19}$ S. Westermann, M. Kreitschmann, W. Pyckhout-Hintzen, D. Richter, E. Straube, B. Farago, and G. Goerigk, Macromolecules 32, 5793 (1999).

${ }^{20}$ A. Botti, W. Pyckhout-Hintzen, D. Richter, V. Urban, E. Straube, and J. Kohlbrecher, Polymer 44, 7505 (2003).

${ }^{21}$ S. Westermann, W. Pyckhout-Hintzen, D. Richter, E. Straube, S. Egelhaaf, and R. May, Macromolecules 34, 2186 (2001).

${ }^{22}$ J. Berriot, D. Lequeu, H. Montes, and H. Pernot, Polymer 43, 6131 (2002).

${ }^{23}$ P. Flory, Principles of Polymer Chemistry (Cornell University Press, Ithaca, New York, 1953).

${ }^{24}$ V. Urban, thesis, University of Munster, 1995.

${ }^{25}$ G. Beaucage, J. Appl. Crystallogr. 29, 134 (1996). 